\title{
MEMS micromirror based light sheet generator for biomedical imaging
}

\author{
Ralf Bauer and Deepak Uttamchandani \\ Centre for Microsystems and Photonics, University of Strathclyde, Glasgow, UK
}

\begin{abstract}
Two MEMS micromirrors with resonant or static actuation are used as a MEMS enabled light sheet generator, with light sheet dimensions of $3.5 \mu \mathrm{m}$ by $550 \mu \mathrm{m}$ and offset positioning of $150 \mu \mathrm{m}$ in the focal plane.
\end{abstract}

Keywords-MEMS, micromirror, electrostatic and electrothermal actuation, lightsheet, biomedical imaging

\section{INTRODUCTION}

The use of light sheets as sculptured excitation for biomedical imaging has had growing interest over the last decade, following the demonstration of selective plane illumination microscopy by Huisken et al. [1]. Two general concepts are commonly used. The creation of a light sheet through the use of cylindrical lenses, with a compact concept being demonstrated recently [2], or the use of scanned single point laser beams [3]. The advantages of using scanning systems are the extra flexibility for tailored dimensions of the light sheet and the potential for easier synchronization control. The integration of MEMS optical scanning micromirrors allows the prospect of further miniaturization together with added functionality through positioning control in the 3D sample space.

In this paper, a concept for a miniaturized light sheet illumination beam path is presented, incorporating two MEMS micromirrors as beam shaping elements. The two single crystal silicon micromirrors are used for light sheet generation and its positioning in the focal plane. They have electrostatic (ES) comb-drive actuation for the 1D scan of a laser point source and electrothermal (ET) actuation for $2 \mathrm{D}$ tip-tilt control to position the light sheet in the focal plane [4]. The illumination system uses a fiber-coupled 488nm Argon-Ion laser as source and is experimentally evaluated for its spatial characteristics in the focal plane.

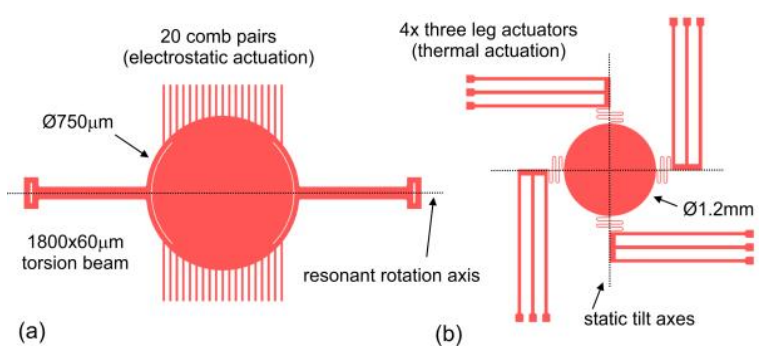

Fig.1: Schematic of (a) the resonant scanning mirror and (b) the static positioning mirror.

This work was supported by the Royal Academy of Engineering under the Engineering for Development Research Fellowship scheme (RF1516\15।8).

\section{MICROMIRROR DESIGN \& CHARACTERIZATION}

Both micromirrors are fabricated using a commercial silicon-on-insulator multi-user process (SOIMUMPs) run by MEMSCAP Inc. The micromirror designs (see Fig. 1) use a silicon device layer of $25 \mu \mathrm{m}$ thickness for the ES comb-drive actuated scanning mirror and a $10 \mu \mathrm{m}$ thickness for the ET actuated positioning mirror. Both are fully backside released through a deep reactive ion etch step. The scanning mirror has an active mirror surface diameter of $700 \mu \mathrm{m}$, torsion springs of $550 \mu \mathrm{m}$ length and $60 \mu \mathrm{m}$ width and 20 comb-pairs with $10 \mu \mathrm{m}$ thickness and $6 \mu \mathrm{m}$ electrode gap placed at the edges of the mirror surface. Spring connections are placed on the edge of the mirror surface and the substratetorsion beam connection to reduce stress and dynamic surface deformation of the mirror surface. The mirror surface radius of curvature (ROC) was measured as $0.4 \mathrm{~m}$. The positioning micromirror has an active mirror diameter of $1.2 \mathrm{~mm}$ with 4 actuators placed symmetrically around the circumference. Each actuator consists of 3 beams with width of $40 \mu \mathrm{m}$ and length of $1.8 \mathrm{~mm}$ (for horizontal tilt) and $1.7 \mathrm{~mm}$ (for vertical tilt). This enables decoupling of the resonance modes of both axes. Out-of-plane movement of the actuator tip is achieved through Joule Heating
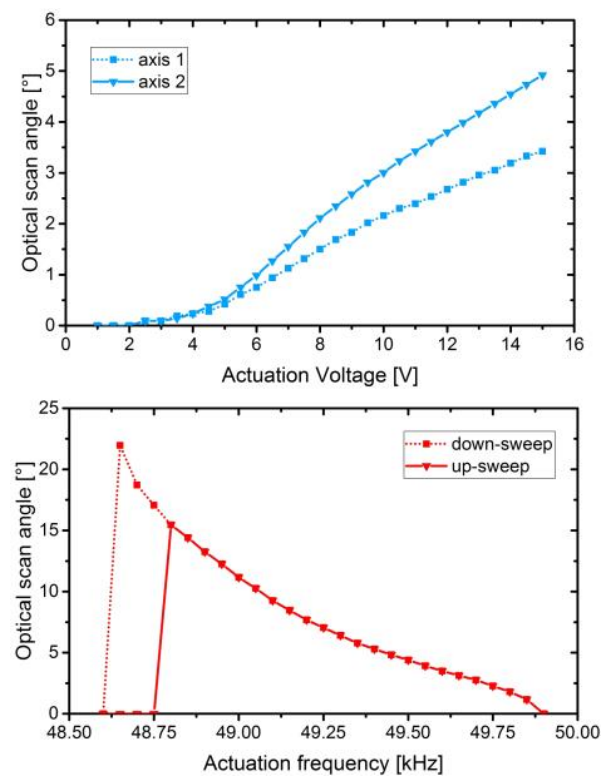

Fig. 2: Tilt angle vs voltage for static mirror (top) and tilt angle vs frequency at $150 \mathrm{Vpp}$ for resonant scanning mirror (bottom). 


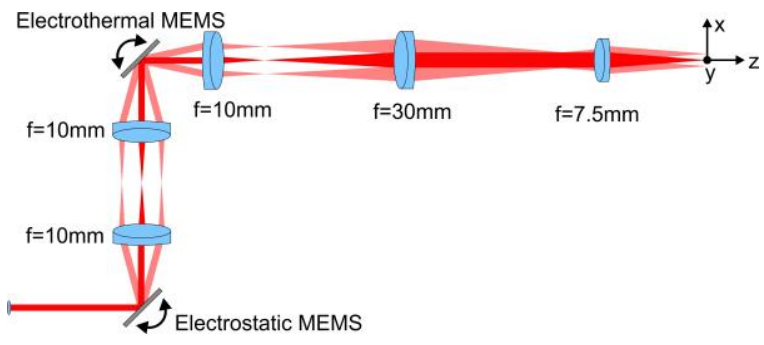

Fig. 3: Optical setup of light sheet illumination path.

resulting from a current flow through the outer actuator beams while the non-actuated central beam acts as mechanical constraint directing the heat induced deformation. Serpentine springs connect each actuator to the mirror surface. The micromirror had a surface ROC of $0.07 \mathrm{~m}$. Static displacement can be achieved through DC voltage signals on the actuators.

The experimental characterization of both mirrors is shown in Fig. 2. The ET static positioning mirror (top) is actuated with a DC voltage between $0-15 \mathrm{~V}$, with the resulting optical tilt angles reaching $4.9^{\circ}$ and $3.4^{\circ}$ for $15 \mathrm{~V}$ actuation. The ES resonant scanning mirror (Fig. 2, bottom) is actuated with a square wave drive voltage with $150 \mathrm{Vpp}$ and $0 \mathrm{~V}$ minimum level. A first harmonic drive scheme is used, resulting in the actuation frequency being double the mechanical movement frequency of the mirror. The resulting angular response shows a spring softening behavior, with hysteresis occurring between $48.65 \mathrm{kHz}$ and $48.8 \mathrm{kHz}$ and a maximum full optical scan angle of $22^{\circ}$. The occurring hysteresis leads to an applied actuation frequency of $48.8 \mathrm{kHz}$ with an optical scan angle of $15.5^{\circ}$.

\section{MEMS LIGHTSHEET GENERATION}

The optical setup to create the scanned light sheet with $2 \mathrm{D}$ positioning capability is shown in Fig. 3. A fiber-coupled 488nm Argon-Ion laser with $1.5 \mathrm{~mW}$ output power is used as excitation source for the light sheet generator. The collimated beam is scanned by the ES micromirror with an incident beam diameter of $500 \mu \mathrm{m}$ and imaged on to the ET positioning mirror with a $4 \mathrm{f}$ system comprising of two $10 \mathrm{~mm}$ achromatic doublet lenses (Thorlabs AC050-010-A). The scan line reflected on the ET mirror is collimated with a further $10 \mathrm{~mm}$ achromatic doublet lens, with the scanned spot being magnified by a 1:3 telescope to a beam diameter of $1.5 \mathrm{~mm}$. The $30 \mathrm{~mm}$ achromatic doublet lens (Thorlabs AC127-030-A) used as second telescope lens collimates the scanned beam. A $7.5 \mathrm{~mm}$ achromatic doublet lens (Thorlabs AC050-008-A) is used as telecentric excitation lens for the system, resulting in a calculated point spread function FWHM of $2.5 \mu \mathrm{m}$ at the focal point and a light sheet height of $670 \mu \mathrm{m}$ for a scan angle of $15.5^{\circ}$. The optical parameters were simulated using Optenso's Optalix ray trace software, leading to a working distance after the focusing lens of $5.13 \mathrm{~mm}$. The overall optical

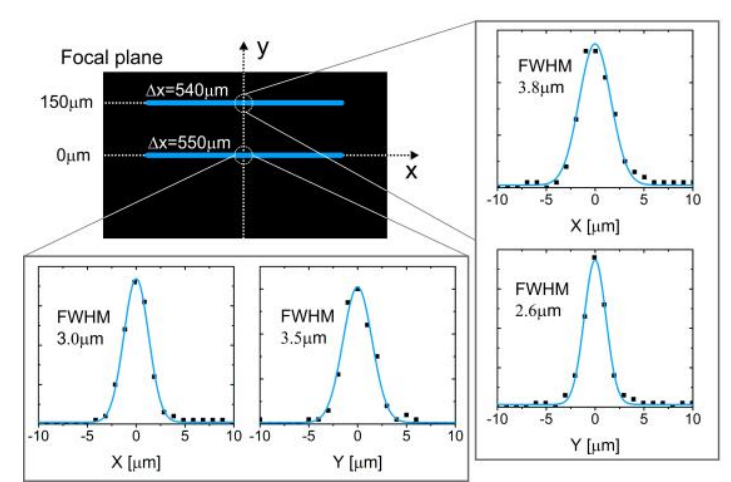

Fig. 4: Light sheet dimensions in focal plane at the center and at a ET-MEMS position of $15 \mathrm{~V}_{\mathrm{DC}}$.

footprint between fiber-collimation and focal point is $13.5 \mathrm{~cm} \times 5 \mathrm{~cm}$.

The experimental setup of the system collimated the laser beam output after the ES micromirror while taking into account its ROC. The measured light sheet dimensions for a scan movement with $24.4 \mathrm{kHz}$ and $15.5^{\circ}$ are shown for the center position and at positioning on the maximum y-axis (Fig. 4). The light sheet dimensions were measured using a knife-edge with a $1 \mu \mathrm{m}$ step size translation stage. The resulting light sheet has a FWHM width of $3.5 \pm 1 \mu \mathrm{m}$ at the center and $2.6 \pm 1 \mu \mathrm{m}$ at a position $150 \mu \mathrm{m}$ above the center line. The light sheet length was measured at $550 \pm 5 \mu \mathrm{m}$ and $540 \pm 5 \mu \mathrm{m}$ for these positions. An intensity increase can be seen at the edges of the light sheet due to the slower scan speed at the change of the MEMS resonant movement direction. The Rayleigh range of the light sheet is therefore around $60 \mu \mathrm{m}$, leading to a confocal parameter of $120 \mu \mathrm{m}$.

\section{CONCLUSION}

The initial concept and characterization of a MEMS enabled light sheet generator is presented, using two micromirrors with ES and ET actuation for the light sheet generation and positioning. Scanned light sheets with width of $3.5 \mu \mathrm{m}$ and length of $550 \mu \mathrm{m}$ are generated and their positioning in the focal plane with up to $150 \mu \mathrm{m}$ offset is demonstrated. This concept is the first step to a fully MEMS controlled miniaturized light sheet microscope.

\section{REFERENCES}

[1] J. Huisken et al.,"Optical sectioning deep inside live embryos by selective plane illumination microscopy.," Science, vol. 305, no. 5686, pp. 1007-1009, Aug. 2004.

[2] Z. Guan et al., "Compact plane illumination plugin device to enable light sheet fluorescence imaging of multi-cellular organisms on an inverted wide-field microscope," Biomed. Opt. Express, vol. 7, no. 1, pp. 194-208, 2016.

[3] P. J. Keller et al., "Reconstruction of zebrafish early embryonic development by scanned light sheet microscopy.," Science, vol. 322, no. 5904, pp. 1065-1069, Nov. 2008.

[4] A. Paterson et al., "Spectral and temporal control of Qswitched solid-state lasers using intracavity MEMS," in SPIE Photonics West 2016, 2016, p. 97261 U. 